\title{
Electric field concentration in hydrogel-elastomer devices
}

\author{
Vaicekauskaite, Justina; Yang, Canhui; Skov, Anne Ladegaard; Suo, Zhigang
}

\section{Published in:}

Extreme Mechanics Letters

Link to article, DOI:

10.1016/j.eml.2019.100597

Publication date:

2020

Document Version

Peer reviewed version

Link back to DTU Orbit

Citation (APA):

Vaicekauskaite, J., Yang, C., Skov, A. L., \& Suo, Z. (2020). Electric field concentration in hydrogel-elastomer devices. Extreme Mechanics Letters, 34, [100597]. https://doi.org/10.1016/j.eml.2019.100597

\section{General rights}

Copyright and moral rights for the publications made accessible in the public portal are retained by the authors and/or other copyright owners and it is a condition of accessing publications that users recognise and abide by the legal requirements associated with these rights.

- Users may download and print one copy of any publication from the public portal for the purpose of private study or research.

- You may not further distribute the material or use it for any profit-making activity or commercial gain

- You may freely distribute the URL identifying the publication in the public portal

If you believe that this document breaches copyright please contact us providing details, and we will remove access to the work immediately and investigate your claim. 


\section{Journal Pre-proof}

Electric field concentration in hydrogel-elastomer devices

Justina Vaicekauskaite, Canhui Yang, Anne Ladegaard Skov, Zhigang Suo

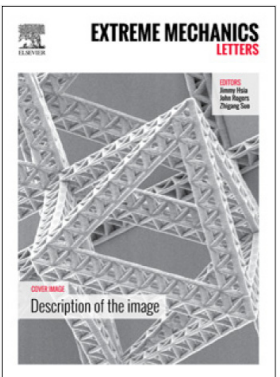

PII:

S2352-4316(19)30265-2

DOI: $\quad$ https://doi.org/10.1016/j.eml.2019.100597

Reference: $\quad$ EML 100597

To appear in: Extreme Mechanics Letters

Received date : 19 June 2019

Revised date : 29 October 2019

Accepted date : 29 October 2019

Please cite this article as: J. Vaicekauskaite, C. Yang, A.L. Skov et al., Electric field concentration in hydrogel-elastomer devices, Extreme Mechanics Letters (2019), doi:

https://doi.org/10.1016/j.eml.2019.100597.

This is a PDF file of an article that has undergone enhancements after acceptance, such as the addition of a cover page and metadata, and formatting for readability, but it is not yet the definitive version of record. This version will undergo additional copyediting, typesetting and review before it is published in its final form, but we are providing this version to give early visibility of the article. Please note that, during the production process, errors may be discovered which could affect the content, and all legal disclaimers that apply to the journal pertain.

(C) 2019 Published by Elsevier Ltd. 


\title{
Electric Field Concentration in Hydrogel-Elastomer Devices
}

$$
\text { Justina Vaicekauskaite, ,, }{ }^{1,2} \text { Canhui Yang,,3 Anne Ladegaard Skov,,** Zhigang Suo, }{ }^{2, *}
$$

${ }^{1}$ The Danish Polymer Centre, Department of Chemical and Biochemical Engineering, Technical University of Denmark, Lyngby, Denmark;

${ }^{2}$ John A. Paulson School of Engineering and Applied Sciences, Kavli Institute for Bionano Science and Technology, Harvard University, MA, 02138, USA;

3 Department of Mechanics and Aerospace Engineering, Southern University of Science and Technology, Shenzhen, Guangdong 518055, PR China;

E-mails: al@kt.dtu.dk, suo@seas.harvard.edu

\begin{abstract}
Hydrogels and elastomers are being integrated to make stretchable, transparent, electromechanical devices. In such a device, a dielectric elastomer functions as an electric insulator, and a salt-containing hydrogel functions as an electric conductor. Here we report several experimental observations associated with electric field concentration along the edges of the hydrogels. We apply cyclic voltage to a large number of samples, and record the numbers of cycles when the elastomer suffers electric breakdown. In most samples, the elastomer breaks down at the edges of the hydrogels. Before the elastomer breaks down, we observe salting out, localized heating, and plasma along the edges of the hydrogels. These observations are consistent with the hypothesis that the concentrated electric field at the edges of the hydrogels breaks down the air when the elastomer is intact. Remarkably, the breakdown of air makes the electric field less concentrated, and protects the elastomer. When the sample is coated with an elastomer, air no longer breaks down, but the elastomer breaks down at a reduced applied voltage. We discuss the significance of these observations in applications.
\end{abstract}

Keywords: hydrogel-elastomer devices; dielectric elastomers; hydrogels; electric field concentration; localized heating; salting out. 


\section{Introduction}

When a membrane of a dielectric elastomer sandwiched between two compliant electrodes is subject to a voltage, charges of opposite polarities accumulate on two sides of the elastomer, resulting in the reduction of thickness and expansion of area (1). This electromechanical transduction has a unique combination of attributes, such as large deformation, fast response, high efficiency, low cost, and light weight (2), and has enabled many devices $(3-5)$. Examples include prosthetic devices $(3,6)$, haptic devices $(7,8)$, soft robots $(1,6,9)$, tunable optics (10,11), sensors (12), and energy harvesters $(1,13)$.

Hydrogels have recently been used as the compliant electrodes (10). A hydrogel is an aggregate of water molecules and a sparse polymer network. Water molecules make the hydrogel liquid-like and optically transparent, while the polymer network makes the hydrogel solid-like and elastically stretchable. Furthermore, the water molecules in the hydrogel can host ions to achieve conductivity up to $10 \mathrm{~S} / \mathrm{m}$ (14). The integration of hydrogels and elastomers has enabled many devices called hydrogel ionotronics (15) or stretchable ionics (16). Examples include transparent loudspeaker (10), ionic skin (12), artificial axon (17), stretchable electroluminescence $(18,19)$, stretchable touchpad $(20)$, and transparent membrane for active noise cancelation (21). In these devices, hydrogels function as stretchable, transparent, ionic conductors, while elastomers function as stretchable, transparent dielectrics.

These promising demonstrations raise an immediate question: what is the reliability of hydrogel-elastomer devices under high voltage over many cycles? Here we report several observations associated with electric field concentration along the edges of the hydrogels. We make each sample by sandwiching a membrane of dielectric elastomer between two membranes of salt-containing hydrogels. When the two hydrogels are subject to a high voltage, the edges of the hydrogels concentrate electric field. We apply cyclic voltage of prescribed amplitude and frequency to a large number of samples, and record the numbers of cycles when the elastomer suffers electric breakdown. In most samples, the elastomer breaks down at the edges of the 
hydrogels (i.e. the regions outside of the area covered with electrode). Before the elastomer breaks down, we observe salting out, localized heating, and plasma along the edges of the hydrogels. These observations support the hypothesis that air breaks down when the elastomer is intact. When the device is coated with an elastomer, air no longer breaks down, but the elastomer breaks down at a reduced applied voltage. We discuss the significance of these observations for hydrogel-elastomer devices.

\section{Results and discussion}

We fabricate a device by sandwiching a layer of elastomer between two layers of hydrogels (Fig. 1a). The elastomer is prestretched and fastened on a rigid frame. The two hydrogels are connected to top and bottom metallic electrodes fixed on the rigid frame. The hydrogels containing ions are electric conductors, and the elastomer is an electric insulator. When a voltage is applied between the two metallic electrodes, electric field vanishes in the hydrogels, but builds up in the elastomer and air (Fig. 1b). Within the area covered by the hydrogels, the electric field lines are straight and uniform in the elastomer, and are negligible in the air. At the edges of the hydrogels, the electric field lines are curved and concentrated, both in the elastomer and in the air. The electric field lines are perpendicular to the surface of the conductors, i.e., the hydrogels.

We use a polydimethylsiloxane (PDMS) elastomer as the stretchable transparent dielectric, and a polyacrylamide (PAAm) hydrogel containing lithium chloride ( $\mathrm{LiCl}$ ) as the stretchable transparent conductor. $\mathrm{LiCl}$ is the most hydroscopic salt used in hydrogel-elastomer devices (14). Hydrogels are connected to voltage source through top and bottom electrodes, which are aluminum foils. The rigid frame is made of acrylic sheet. Because both the PDMS elastomer and the PAAm hydrogel are transparent, the device is also transparent (Fig. 1c). The diameter of the stretched elastomer film is $5 \mathrm{~cm}$ and the diameter of PAAm hydrogel is $2 \mathrm{~cm}$. Unless otherwise specified, the thickness of the unstretched elastomer is $250 \mu \mathrm{m}$. After an equal 
biaxial stretch of 1.2, the thickness reduces to $\sim 173 \mu \mathrm{m}$. The thickness of PAAm hydrogel is $500 \mu \mathrm{m}$.

a)

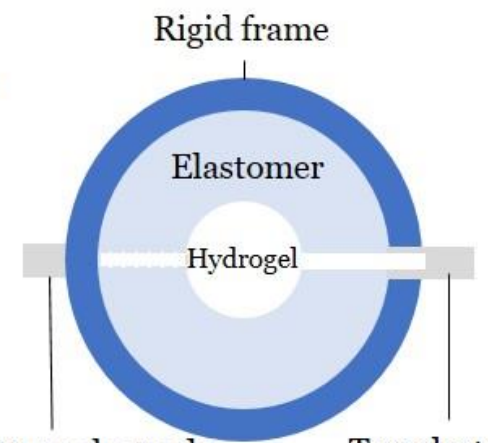

Bottom electrode

Top electrode
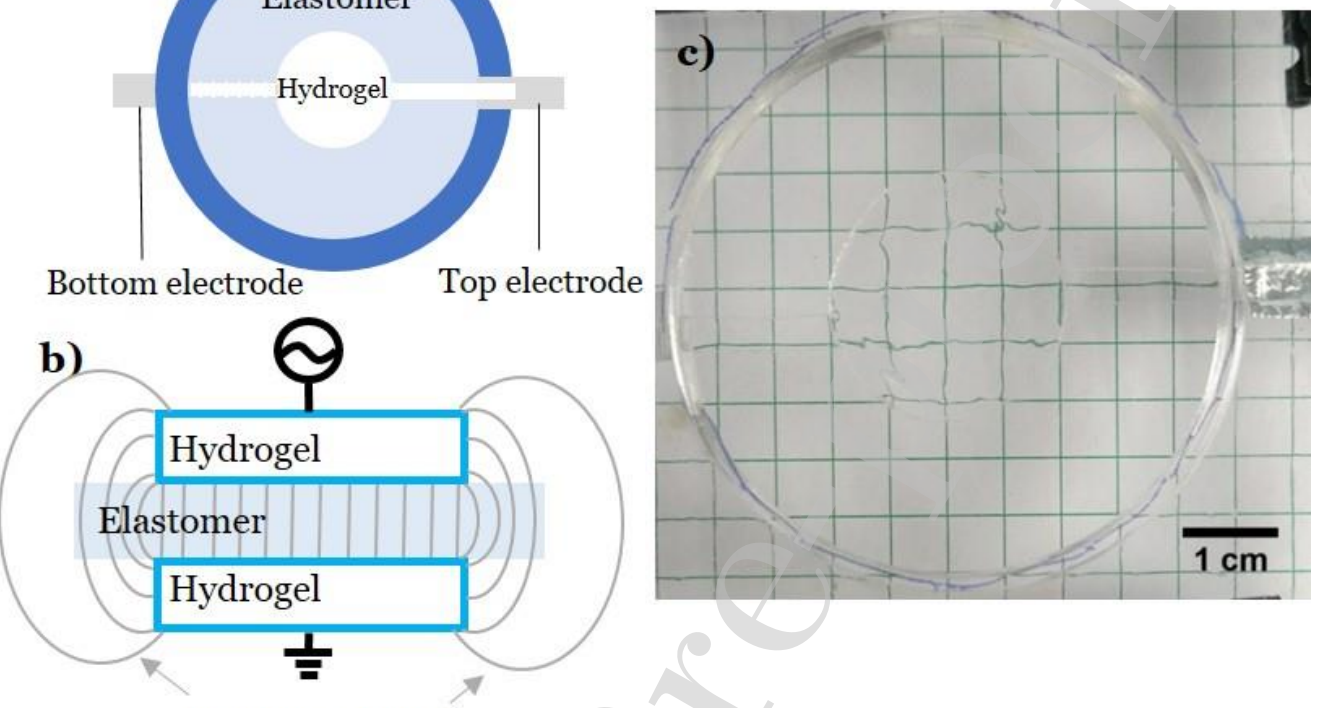

Electric field lines

Figure 1. Experimental set up. a) Schematic top view of a hydrogel-elastomer device. A membrane of a dielectric elastomer is prestretched and fastened onto a rigid frame. Two circular membranes of hydrogels are attached on both sides of the elastomer and are connected, via two lines of hydrogels, to top and bottom metallic electrodes fixed on the rigid frame. b) Schematic side view of the device. When a voltage is applied between the two metallic electrodes, electric field concentrates at the edges of the hydrogels. c) A photo of a hydrogel-elastomer device.

We first study the effect of electric field concentration on the lifetime of the device. We use the PAAm hydrogels containing various concentrations of LiCl. To minimize the effects of ambient humidity, we conduct the experiments inside a chamber and control the interior humidity by placing a tank of $\mathrm{LiCl}$ solution of the same concentration as that in the hydrogel (Fig. S1). The interior humidity is controlled by placing a tank of $\mathrm{LiCl}$ solution of the same concentration as that in the hydrogel. If carbon grease electrode is used, the concentration of $\mathrm{LiCl}$ is fixed at $8 \mathrm{~mol} / \mathrm{L}$. The humidity is approximately $88 \%$ for $1 \mathrm{~mol} / \mathrm{L}, 80 \%$ for $4 \mathrm{~mol} / \mathrm{L}$, $52 \%$ for $8 \mathrm{~mol} / \mathrm{L}$ and $32 \%$ for $12 \mathrm{~mol} / \mathrm{L} \mathrm{LiCl}$ solution. We apply a sinusoidal voltage of frequency 
$1 \mathrm{kHz}$. This frequency is chosen to optimize overall measurement time. The areal strain is less than $5 \%$ and the change of electric field due to areal strain is neglected. We measure the breakdown voltage by ramping up the amplitude of the sinusoidal voltage at a rate of $300 \mathrm{~V} / \mathrm{s}$ speed. Then we apply voltage of the same frequency and a prescribed amplitude to many samples. When breakdown occurs, a small pinhole is observed on the elastomer. Dielectric elastomer is pre-stretched thus sample can tear, but this is not observed in all of the experiments. If the elastomer of a sample breaks down within 10 minutes, we plot the data point in the diagram with the axis of the amplitude of voltage and the number of cycles to breakdown (Fig. 2). If the elastomer of a sample does not break down in 10 minutes, the experiment is then terminated and the result is represented as an arrow in the diagram. Also included in the diagram are data of breakdown measured by applying voltage with ramping amplitude.

Both the breakdown voltage measured using ramping voltage and the number of cycles to breakdown measured using cyclic voltage of prescribed amplitude have large statistical scatter for all concentrations of $\mathrm{LiCl}$. For most samples, the elastomer breaks down at the edges of the hydrogels (94\% for PAAm samples with $1 \mathrm{~mol} / \mathrm{L} \mathrm{LiCl,} 100 \%$ for PAAm samples with $4 \mathrm{~mol} / \mathrm{L} \mathrm{LiCl}, 67 \%$ for PAAm samples with $8 \mathrm{~mol} / \mathrm{L} \mathrm{LiCl}$ and $81 \%$ for PAAm samples with $12 \mathrm{~mol} / \mathrm{L} \mathrm{LiCl)}$. Longer lifetime is obtained at lower voltage. Samples with lower $\mathrm{LiCl}$ concentration tends to have shorter lifetime. The relative humidity is higher when the concentration $\mathrm{LiCl}$ solution is lower (Fig. S1). For comparison, the diagram also includes the data for samples using carbon grease as the compliant electrodes. The lifetime of carbon grease driven devices is also noisy and $97 \%$ devices break down on the edges. These results confirm that the edges of the electrodes (both the hydrogel and the carbon grease) concentrate the electric field, and cause the elastomer to breakdown at the edges of the electrodes. 


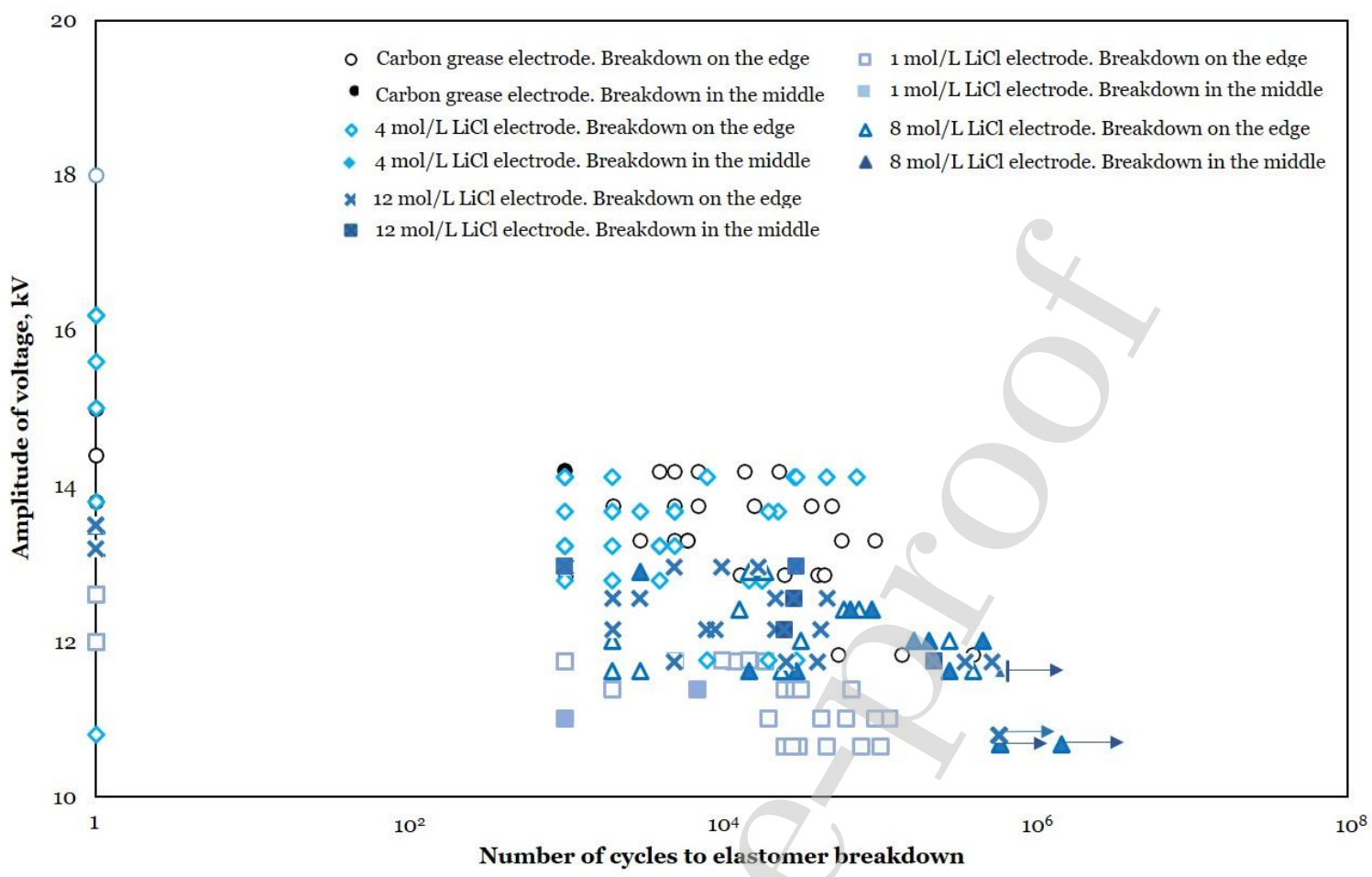

Figure 2. Endurance of hydrogel-elastomer devices. Each data point represents a sample subject to cyclic voltage of prescribed amplitude and frequency, when the elastomer breaks down after some number of cycles. An arrow on a data point means that the sample has not broken down when the measurement is terminated. Unfilled symbols represent the breakdown of the elastomer at the edges of the compliant electrodes, and filled symbols represent the breakdown of the elastomer in the middle of the compliant electrodes. Also included along the vertical axis are the breakdown voltages measured under ramping voltage. This diagram contains the data for a total of 160 samples.

We next study the effect of field concentration before electric breakdown. We observe that the $\mathrm{LiCl}$ in the hydrogels precipitates out during the experiment (Fig. 3a). We call this phenomenon salting out. Before the cyclic voltage is applied, $\mathrm{LiCl}$ fully dissolves in the hydrogels and the hydrogels are transparent. After we apply a sinusoidal voltage of frequency $1 \mathrm{kHz}$ and amplitude $10680 \mathrm{~V}$ for 5 minutes, certain areas of the hydrogels, especially at the edges, turn white due to the precipitation of $\mathrm{LiCl}$. The elastomer around the electrodes after experiment also gets opaque because of appearing cracks on the elastomers. The surrounding cracks might be caused by corona discharge due to the roughness of the hydrogels (22). After 10 minutes of experiment, more pronounced salting out is observed with the precipitation area approaching 
more toward the interior. After the voltage is switched off for 1 hour, the hygroscopic LiCl absorbs water from the ambient and dissolves in the hydrogels again, so that the hydrogels regain transparency.

We use a thermal camera (FLIR TG165) to monitor the device (Fig. 3b). Before experiment, temperature of the device is similar to that of ambient environment. The highest temperature measured is $21.9^{\circ} \mathrm{C}$, approximately the room temperature. After the voltage is applied for 5 minutes, the areas along the edges of the hydrogels show higher temperature than the rest of areas. At this stage, the highest temperature is measured to be $50.1^{\circ} \mathrm{C}$. After 10 minutes, the local temperature further increases with the highest temperature reaching $66.6^{\circ} \mathrm{C}$. Subsequently, we switch off the voltage. After 1 hour, the temperature of the device recovers to room temperature. We conclude that it is the local heating that causes the hydrogels to lose water, which then results in salting out. We verify that the hydrogels containing $8 \mathrm{~mol} / \mathrm{L}$ $\mathrm{LiCl}$ dry out at $65^{\circ} \mathrm{C}$ (Fig. S2). We confirm that local heating also occurs for samples driven by carbon grease (Fig. S3). The oil of carbon grease does not have noticeable drying out. However, for other types of carbon grease, increase in temperature causes the electrodes to flow (Fig. $\mathrm{S} 4 \mathrm{a}$ ). 


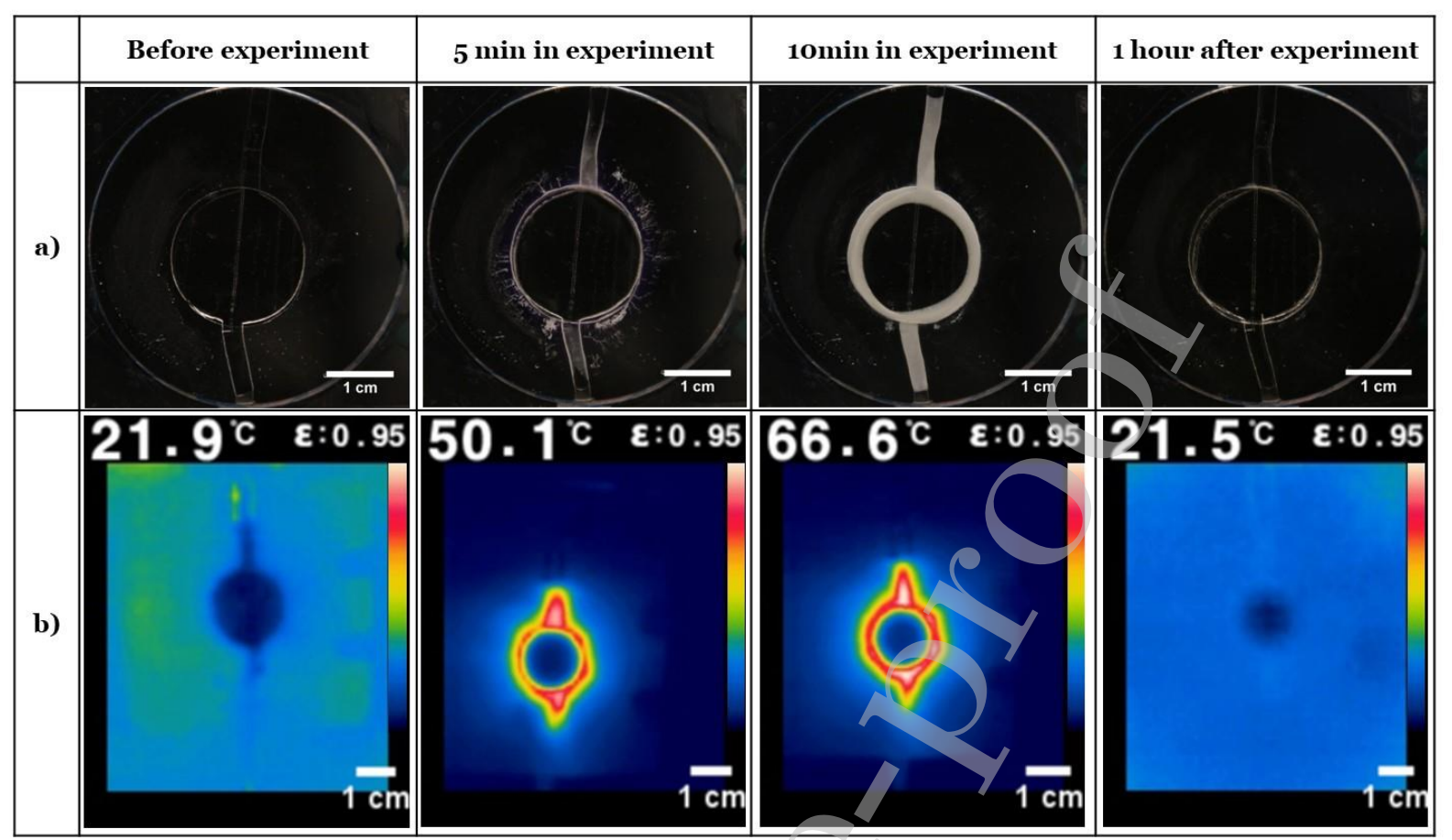

Figure 3. Salting out and localized heating. a) Images showing the evolution of the salting out process of a PDMS driven by PAAm hydrogels containing $8 \mathrm{~mol} / \mathrm{L} \mathrm{LiCl} \mathrm{under} \mathrm{applied} \mathrm{voltage} \mathrm{of}$ amplitude $10680 \mathrm{~V}$ and frequency $1 \mathrm{kHz}$. b) Corresponding thermal images. The highest local temperature reaches $66.6^{\circ} \mathrm{C}$ after 10 minutes of experiment.

We further study the effects of frequency and amplitude of applied voltage on salting out and localized heating. When the amplitude of applied voltage is fixed at $10680 \mathrm{~V}$ and the experimental time is set to be 10 minutes, salting out becomes more and more pronounced as the frequency increases from $0.5 \mathrm{kHz}$ to $1 \mathrm{kHz}$ (Fig. S5). Similarly, higher amplitude of applied voltage leads to higher local temperature thus more pronounced salting out (Fig. S6).

What causes salting out and localized heating? The electric breakdown strength of the air is much smaller than that of the elastomer. We hypothesize that the electric field concentrated at the edges of the hydrogels cause the air breakdown when the elastomer is still intact (Fig. 4a). We test this hypothesis by applying cyclic voltage to samples placed in a dark room. We see purple light along the edges of the hydrogels (Fig. 4b). We surmise that the plasma is caused by air breakdown, which causes salting out and localized heating. We also observe plasma when carbon grease is used as electrodes (Fig. S4b). 
To further test the hypothesis of air breakdown, we coat the hydrogel-elastomer device with a thin layer of PDMS elastomer (Fig. 4c). No plasma is observed prior to elastomer breakdown. The elastomer in coated samples breaks down at a voltage lower than the elastomer in uncoated samples (Fig. 4d). We interpret this experimental finding as follows. It is reasonable to approximate the plasma as an electric conductor, so that the electric field inside the plasma vanishes, and the electric field outside the plasma approaches the plasma perpendicularly. The size scale of the plasma is larger than the thickness of the hydrogel. Once air breaks down, the electric field nearby is limited by the breakdown electric field of the air, which is much below the breakdown field of the elastomer. Consequently, the air breakdown alleviates field concentration, and increases the voltage to breakdown the elastomer. We further vary the thickness of hydrogels between $250 \mu \mathrm{m}$ and $1.5 \mathrm{~mm}$, but the breakdown voltage does not show obvious dependence on the thickness (Fig. 4e). In all cases, when the devices are coated with elastomer, the breakdown voltage of the devices drops significantly. Similar behavior is observed when carbon grease is used. These results further support that the breakdown of air protects the devices.

We note that discrepant observation that encapsulation leads to the decrease of breakdown voltage in dielectric elastomer actuator has been reported in previous study (23). We attribute the discrepancy to two differences. Firstly, salt-containing hydrogel which is ionically conductive and contains a lot of water is used in this work, while electronic conductors (e.g. graphite power) is used in previous work. Secondly, the thickness of hydrogel electrode (500 $\mu \mathrm{m}$ ) is much larger than that of graphite electrode (less then $\sim 10 \mu \mathrm{m}(24)$ ), so that the influence of electric field concentration can be more pronounced. 

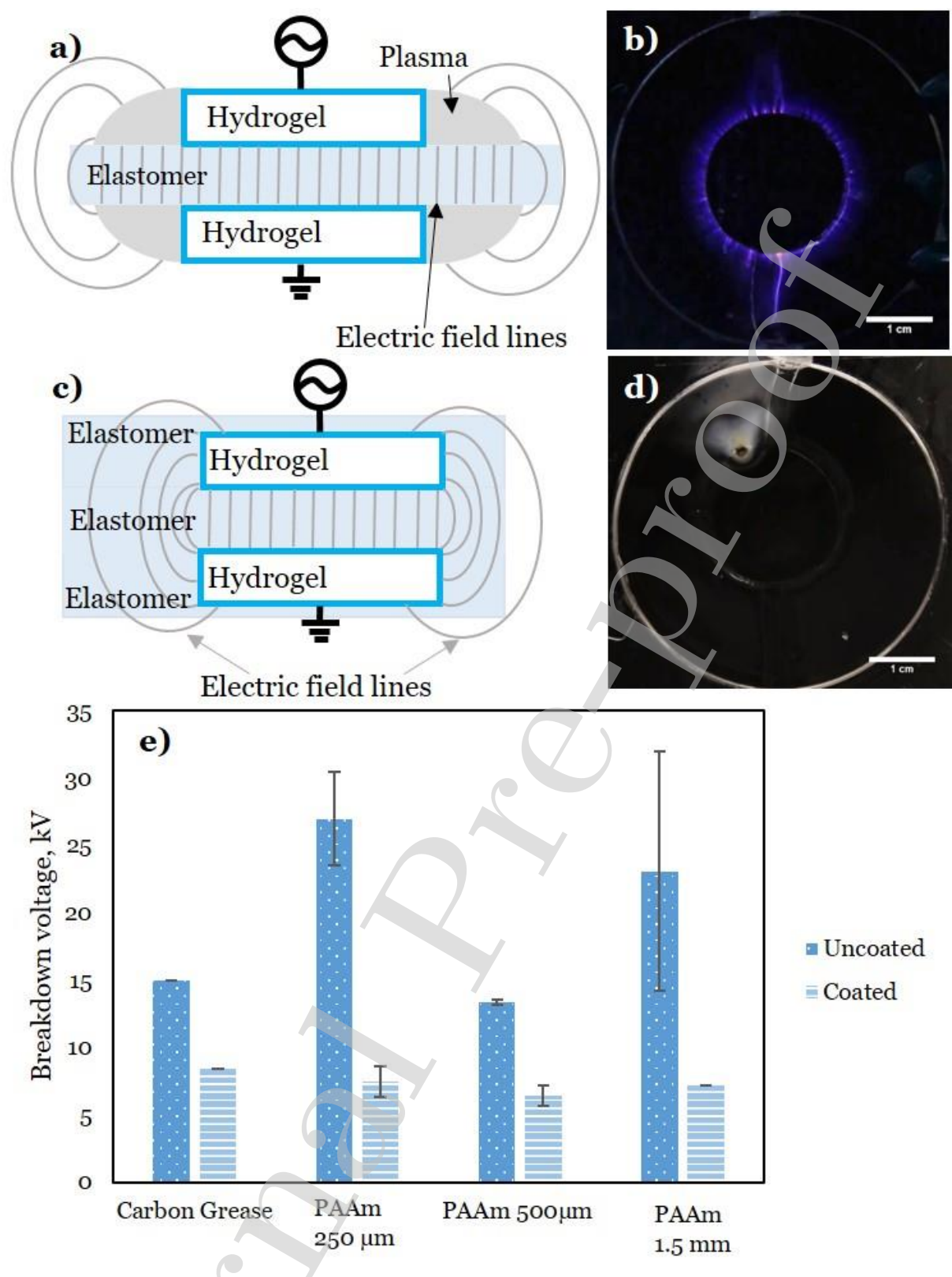

Figure 4. Hydrogel plasma. a) Schematic of an uncoated device. Air breaks down at the edges of hydrogels, creating plasma. b) Subject to cyclic voltage, a plasma of purple light is produced along the edges of the hydrogels. c) Schematic of an elastomer-coated device. Air no longer breaks down, but the electric field concentrates in the elastomer. d) Photo of the device after the elastomer breaks down. All coated samples break down at the edge. e) Comparison of breakdown voltage in coated and uncoated samples using various electrodes. At least 4 samples of one composition are used for each experiment. 


\section{Concluding remarks}

In summary, we have studied the electric field concentration in hydrogel-elastomer devices. We show that the lifetime has large statistical scatter. The field concentration causes the elastomer in the devices to break down mostly at the edges of the hydrogels. Prior to elastomer breakdown, air breaks down along the edges of hydrogels, causing salting out, localized heating, and plasma. Given that $\mathrm{LiCl}$ is among the best hygroscopic salt used for hydrogels, one should take into account of salting out and localized heating when the amplitude of applied voltage is large, frequency of applied voltage is high, or the work time is long. Compared to hydrogels, ionogels containing ionic liquid can operate stably at high temperature $\left(>100^{\circ} \mathrm{C}\right)$ or even under vacuum $(25,26)$, providing an alternative to achieve soft, stretchable and transparent actuators without the concerns of drying out. Electric field concentration is generic for all electrical devices and has numerous well-established applications, including lightening rod, ozone production, and sanitization of water. Recently, electric field concentration has enabled a new concept of airplane with no moving parts and solid-state propulsion (27). The field concentration in hydrogel-elastomer devices opens doors for new applications. For example, compared to conventional electrical devices, hydrogel-elastomer devices are soft and biocompatible. The field concentration of these devices can be used for tissue engineering such as non-invasive cell manipulation and on-demand reconstruction of artificial tissues (28). In addition, the amplification of electric field reduces the working voltage in voltage-driven devices, e.g. electroluminescent displays and liquid-crystal displays (29). Furthermore, localized heating and the salting out are built-in indicators for the safe operation of the devices.

\section{Acknowledgements}

The work at Harvard was supported by NSF MRSEC (DMR-14-20570). J. Vaicekauskaite was supported by Otto Mønsteds Fond and Idella Foundation as a visiting student at Harvard for 
6 months. The Independent Research Fund Denmark is acknowledged for funding the project. C. Yang acknowledge the start-up funding from Shenzhen and SUSTech (Yo1326119 \& Yo1326219).

\section{Experimental section}

\section{Synthesis of PAAm hydrogels}

$2 \mathrm{~mol} / \mathrm{L}$ acrylamide (AAm, Sigma-Aldrich, A8887) solution is prepared. For every $1 \mathrm{ml}$ of the solution, we add $4 \mu \mathrm{L} 0.1 \mathrm{~mol} / \mathrm{L}$ crosslinking agent, N,N' methylenebisacrylamide (MBAA, Sigma-Aldrich, 146072), and $20 \mu \mathrm{L} 0.1 \mathrm{~mol} / \mathrm{L}$ photoinitiator, $\alpha$-ketoglutamate acid ( $\alpha$-keto, Sigma-Aldrich, 75890). Lithium chloride ( $\mathrm{LiCl}$, Sigma-Aldrich, 746460) is added to yield precursors with different $\mathrm{LiCl}$ concentrations: $1 \mathrm{~mol} / \mathrm{L}, 4 \mathrm{~mol} / \mathrm{L}, 8 \mathrm{~mol} / \mathrm{L}$ and $12 \mathrm{~mol} / \mathrm{L}$. The precursor is cast in $160 \times 160 \times 0.5 \mathrm{~mm}^{3}$ glass mold and cured for 1 hour under a $15 \mathrm{~W}$ bench UV Lamp (XX-15, UVP) at a dose rate of $30 \mathrm{~mW} / \mathrm{cm}^{2}$ at $365 \mathrm{~nm}$ in room temperature. Circular shaped electrodes with thin lines are cut out from the cured hydrogel film, using a laser cutter (Helix 75W laser cutter, LZR-010).

\section{Preparation of elastomer}

Two parts of silicone rubber (Sylgard 184, Dow Corning), base and curing agent, are mixed in recommended ratio of 10:1. The mixture is cast into a $100 \times 100 \times 0.250 \mathrm{~mm}^{3} \mathrm{mold}$ and cured for 4 hours, at 65 degree Celsius. After curing, the elastomer film is removed from the mold, stretched by 20\% strain biaxially, and fixed on a rigid acrylic frame (Fig. 1).

\section{Humidity control}

We make a humidity chamber to control the relative humidity during the experiments. We cut acrylic sheets using laser cutter and glue them to form a chamber of dimensions $30 \times 16 \times 15 \mathrm{~cm}^{3}$. Humidity equilibrium in the chamber is tested by placing a tank of $100 \mathrm{~mL}$ LiCl solution inside of the chamber (Fig. S1d) and monitoring humidity change in 24 hours 
using a humidometer. The lifetime measurements are carried out when the humidity inside the chamber reaches equilibrium.

\section{Fabrication of devices}

A device is made by placing a layer of elastomer between two layers of electrodes. Two types of electrodes are used: carbon grease (CG, MG Chemicals, \#846-80G), which is stenciled on both sides of elastomer, and polyacrylamide (PAAm) hydrogel containing LiCl. Electrodes are linked through thin electrode lines to two aluminum foils placed on top and bottom of the acrylic frame. For the coated samples, we dip coat the electrodes with the precursor of silicone elastomer (Sylgard 184, Dow Corning), base and curing agent are mixed in recommended ratio of 10:1, and cure at room temperature overnight. During testing, the two aluminum foils are connected to a high voltage amplifier (Trek, 30/20A), which amplifies the input voltage generated by a waveform generator (KEYSIGHT, 33500B) with an amplification factor of 3000 .

\section{Thermal and optical images}

Thermal images are taken using Flir TG165 imaging IR thermometer with $80 \times 60$ resolution and accuracy of $\pm 1.5 \%$. When a sample is subject to cyclic voltage, the thermal camera is pointed at the hottest spot of the sample. The number at the left top indicates the hottest temperature in that thermal image. Optical images are taken using a digital camera (Canon Ds126321). 


\section{References}

1. Pelrine RE, Kornbluh RD, Joseph JP. Electrostriction of Polymer Dielectrics with Compliant Electrodes as a Means of Actuation. Sensors Actuators, A Phys. 1998;64:77-85.

2. Carpi F, De Rossi D, Kornbluh R, Pelrine R, Sommer-Larsen P. Dielectric Elastomers as Electromechanical Transducers: Fundamentals, Materials, Devices, Models and Applications of an Emerging Electroactive Polymer Technology. 2011. 51-68 p.

3. Pelrine RE, Kornbluh RD, Pei Q, Joseph J. High-Speed Electrically Actuated Elastomers with Strain Greater Than 100\%. Science. 2000;287:836-40.

4. Anderson IA, Gisby TA, Mckay TG, Brien BMO, Calius EP. Multi-functional Dielectric Elastomer Artificial Muscles for Soft and Smart Machines. J Appl Phys. 2012;112(041101):1-20.

5. Brochu P, Pei Q. Advances in Dielectric Elastomers for Actuators and Artificial Muscles. Macromol Rapid Commun. 2010;31:10-36.

6. Acome E, Mitchell SK, Morrissey TG, Emmett MB, Benjamin C, King M, et al. Hydraulically Amplified Self-healing Electrostatic Actuators with Muscle-like Performance. Science. 2018;359:61-5.

7. Pyo D, Ryu S, Kyung K, Yun S, Kwon D. High-pressure Endurable Flexible Tactile Actuator Based on Microstructured Dielectric Elastomer. Appl Phys Lett. 2018;112(061902):1-5.

8. Tiwari N, Rajput M, Chien N, Mathews N. Highly Transparent and Integrable Surface Texture Change Device for Localized Tactile Feedback. Small J. 2018;14(1702312):1-9.

9. Jordi C, Michel S, Fink E. Fish-like Propulsion of an Airship With Planar Membrane Dielectric ElastomerActuators. Bioinspiration and Biomimetics. 2010;5(026007):1-9.

10. Keplinger C, Sun J, Foo C, Rothemund P, Whitesides GM, Suo Z. Stretchable, Transparent, Ionic Conductors. Science. 2013;341(6149):984-7.

11. Jin B, Ren H. Position-movable Lens Driven by Dielectric Elastomer Actuator. Opt Eng. 2016;55(7):1-7.

12. Sun J, Keplinger C, Whitesides GM, Suo Z. Ionic Skin. Adv Mater. 2014;26:7608-14.

13. Moretti G, Papini GPR, Righi M, Forehand D, Ingram D, Vertechy R, et al. Resonant Wave Energy Harvester Based on Dielectric Elastomer Generator. Smart Mater Struct. 2018;27(035015):1-14.

14. Bai Y, Chen B, Xiang F, Zhou J, Wang H, Suo Z. Transparent Hydrogel with Enhanced Water Retention Capacity by Introducing Highly Hydratable Salt. Appl Phys Lett. 2014;105(151903):1-5. 
15. Yang C, Suo Z. Hydrogel Ionotronics. Nat Rev Mater. 2018;

16. Lee HR, Kim CC, Sun JY. Stretchable Ionics - A Promising Candidate for Upcoming Wearable Devices. Adv Mater. 2018;30(42):1-15.

17. Yang C, Chen B, Jing J, Hai J, Zhou J, Mei Y, et al. Ionic Cable. Extrem Mech Lett. 2015;3:59-65.

18. Shepherd R, Larson C, Peele B, Li S, Robinson S, Totaro M, et al. Highly Stretchable Electroluminescent Skin for Optical Signaling and Tactile Sensing. Science. 2016;351(6277):1071-4.

19. Yang CH, Chen B, Zhou J, Chen YM, Suo Z. Electroluminescence of Giant Stretchability. Adv Mater. 2015;3(00):1-4.

20. Kim CC, Lee HH, Oh KH, Sun JY. Highly stretchable, transparent ionic touch panel. Science. 2016;353(6300):682-7.

21. Rothemund P, Morelle XP, Jia K, Whitesides GM, Suo Z. A Transparent Membrane for Active Noise Cancelation. Adv Funct Mater. 2018;1800653(28):1-8.

22. Hillborg H, Gedde UW. Hydrophobicity Recovery of Polydimethylsiloxane After Repeated Exposure to Corona Discharges. Influence of Crosslink Density. Conf Electr Insul Dielectr Phenom. 1999;751-5.

23. La TG, Lau GK. Very high dielectric strength for dielectric elastomer actuators in liquid dielectric immersion. Appl Phys Lett. 2013;102(19).

24. Thanh-Giang La, Gih-Keong Lau, Li-Lynn Shiau and AW-YT. Muscle-like high-stress dielectric elastomer actuators with oil capsules. Smart Mater Struct. 2014;23(10):1-10.

25. Le Bideau J, Viau L, Vioux A. Ionogels, ionic liquid based hybrid materials. Chem Soc Rev. 2011;40(2):907-25.

26. Chen B, Lu JJ, Yang CH, Yang JH, Zhou J, Chen YM, et al. Highly stretchable and transparent ionogels as nonvolatile conductors for dielectric elastomer transducers. ACS Appl Mater Interfaces. 2014;6(10):7840-5.

27. Xu H, He Y, Strobel KL, Gilmore CK, Kelley SP, Hennick CC, et al. Flight of an aeroplane with solid-state propulsion. Nature. 2018;563(7732):532-5.

28. Markx GH. The use of electric fields in tissue engineering: A review. Organogenesis. 2008;4(1):11-7.

29. Yang CH, Zhou S, Shian S, Clarke DR, Suo Z. Organic liquid-crystal devices based on ionic conductors. Mater Horizons. 2017;4(6):1102-9. 


\section{Conflict of Interest and Authorship Conformation Form}

We confirm that:

- All authors have participated in (a) conception and design, or analysis and interpretation of the data; (b) drafting the article or revising it critically for important intellectual content; and (c) approval of the final version.

- This manuscript has not been submitted to, nor is under review at, another journal or other publishing venue.

- The authors have no affiliation with any organization with a direct or indirect financial interest in the subject matter discussed in the manuscript.

We state that there is no conflict of interest.

Authors: Justina Vaicekauskaite, Canhui Yang, Anne Ladegaard Skov, Zhigang Suo 